\title{
Studies of Anti-Diabetic Effect of Morinda citrifolia Fruit Juice on Alloxan Induced Diabetic Rat
}

\author{
Nitin D. Jadhav, Debi P. Mishra*, Abhinna K. Behera, \\ Sudhir R. Rajurkar and Bhagirath V. Ballurkar
Department of Veterinary Pharmacology and Toxicology, College of Veterinary and Animal Sciences, Parbhani, India
*Corresponding author

\section{A B S T R A C T}

\begin{tabular}{|l|}
\hline Ke y w o r d s \\
Anti-Diabetic, \\
Morinda citrifolia, \\
Fruit Juice, \\
Alloxan Induced \\
Diabetic Rat. \\
\hline Article Info \\
\hline $\begin{array}{l}\text { Accepted: } \\
\text { 15 March } 2017 \\
\text { Available Online: } \\
\text { 10 April } 2017\end{array}$ \\
\hline
\end{tabular}

\section{Keywords}

Anti-Diabetic, Fruit Juice, Alloxan Induced

\section{Introduction}

Diabetes mellitus is a chronic metabolic disorder affecting approximately 5\% of the world's population. It is characterized by dysregulation in the carbohydrate, protein and fat metabolisms caused by the complete or relative insufficiency of insulin secretion and/or insulin action (O' Brien and Granner, 1996). Diabetes mellitus is currently a major life style disease for now a days and has public health importance, because its incidence and prevalence are elevated and increasing, reaching epidemic proportions. Diabetes can be diagnosed by presence of four classical signs that include polyurea, polyphagia, polydipsia and, foremost, hyperglycemia. Diabetes is due to either the pancreas not producing enough insulin or the cells of the body not responding properly to the produced insulin (Gardner and Shoback, 2011).

There are three main types of diabetes mellitus-

(1) Type 1 DM - Results from the body's failure to produce enough insulin, previously 
referred to as insulin dependent diabetes mellitus (IDDM). (2) Type 2 DM - Begins with insulin resistance, a condition in which cells fail to respond to insulin properly, previously referred to as non-insulin dependent diabetes mellitus (NIDDM). (3) Gestational diabetes - Begins when pregnant women without a previous history of diabetes develop a high blood glucose level.

Untreated diabetes can cause many complications. Acute complications include diabetic keto-acidosis and nonketotic hyperosmolar coma (Kitabchi et al., 2009). Serious long term complications include heart disease, stroke, kidney failure, foot ulcers, and damage to the eyes. Diabetes doubles the risk of cardiovascular disease (Sarwar et al., 2010) and $75 \%$ of deaths in diabetics are due to coronary artery disease (O'Gara et al., 2013). Damage to nerves known as diabetic neuropathy, lead to numbness and altered pain sensation. There is a link between cognitive deficit and diabetes. Compared to those without diabetes, those with the disease have a 1.2 to 1.5 fold greater rate of decline in cognitive function (Cukierman et al., 2005). Diabetes mellitus is currently a major public health concern, because its incidence and prevalence are elevated and increasing, reaching epidemic proportions. As at 2013, 382 million people have diabetes worldwide. This is equal to $8.3 \%$ of the adult population (Yuankai and $\mathrm{Hu}, 2014$ ).

Morinda citrifolia Linn (Rubiaceae) also known as Indian mulberry (Noni) is a small evergreen tree. The leaves are 8-10 inches long, oval shaped; dark green and shiny, with deep veins (Rivera et al., 2011). Fruit juice of M. citrifolia is a well known health drink and has various pharmacological properties including anti-diabetic, antioxidant and antiinflammatory (Harada et al., 2010) antitumour activity (Hirazumi and Furusawa, 1999).

\section{Materials and Methods}

\section{Materials}

\section{Experimental animals}

The present study was conducted in 48 Wistar rats of either sex, age 4-6 weeks. The wistar rats of in-house; Animal house facility of College of Veterinary and Animal Sciences, Parbhani were used. Animals were selected after physical and behavioral examination. The Institutional Animals Ethical Committee (IAEC) approved the experimental protocol.

The Wistar rats were kept under constant observation for at least five days before commencement of the experiment. All necessary management procedures were adopted to keep the animals free from stress.

\section{Drugs and chemicals}
Alloxan
The alloxan was used for induction of diabetes in Wistar rats.

$\begin{array}{ll}\text { Chemical name } & : \text { Alloxan } \\ \text { Empirical formula } & : \mathrm{C}_{4} \mathrm{H}_{2} \mathrm{~N}_{2} \mathrm{O}_{4} \cdot \mathrm{H}_{2} \mathrm{O} \\ \text { Batch no } & : 033 / 0326 / \mathrm{P} 1 \\ \text { Vehicle } & : \text { Normal Saline }\end{array}$

\section{Metformin}

Metformin was used as standard oral hypoglycemic drug as is most commonly used oral hypoglycemic agent.

Compound name: Glycomet

Chemical name: Metformin

$\begin{array}{lll} & & \text { Hydrochloride } \\ \text { Batch No } & : & 28006694 \\ \text { Vehicle } & : & \text { Deionised water }\end{array}$




\section{Plant material}

\section{Morinda citrifolia $\mathbf{L}$.}

Botanical name $:$ Morinda citrifolia
$\begin{array}{ll}\text { Family } & \text { : Rubiaceae (coffee family) } \\ \text { Common name: } & \text { Indian Mulberry, } \\ & \quad \text { Great morinda } \\ \text { English } & : \text { Noni } \\ \text { Hindi } & \text { : Bartundi } \\ \text { Sanskrit } & \text { : Ayushka } \\ \text { Marathi } & \text { : Nagakunda } \\ \text { Gujarati } & \text { : Surangi }\end{array}$

Morinda citrifolia L. (Noni) fruits juice

Morinda citrifolia L. fruit juice prepared by the College of Food Science and Technology, MKV, Parbhani was used in the present study.

\section{Methods}

\section{Experimental design}

Table 1 shows experimental design, distribution of different groups and treatment given. Each group contained 12 animals of either sex.

\section{Induction of diabetes}

Out of 48 healthy wistar rats, 36 rats from group II to IV were fasted overnight; diabetes was induced by Intraperitonial administration of alloxan monohydrate (S. D. Fine Chem. Ltd., Mumbai) in physiological saline as 5 percent W/V at the dose rate of $100 \mathrm{mg} / \mathrm{kg}$ body weight. After one week, diabetic status was confirmed in the alloxan administered rats by estimating plasma glucose level $>200$ $\mathrm{mg} / \mathrm{dl}$.

\section{Collection of sample}

Blood samples were collected in aliquots on the 0 day, $14^{\text {th }}$ day $\&$ on the day of animal sacrifice $\left(28^{\text {th }}\right.$ day), from retro-orbital plexus with the help of capillary tube for estimations of blood biochemical parameters.

\section{Parameter studied}

The serum was separated from blood and following parameters were estimated by using serum biochemistry semi-auto analyzer (Model- Chem.7).

Blood glucose (GLU) : (GOD/POD method)

Triglyceride (TG) : (GOD/POD method)

Total ccholesterol (TC) : (CHOD/POD method)

High density lipoprotein (HDL) : (CHOD/POD by HDL precipitating agent)

Low density lipoprotein (LDL) : Total cholesterol minus HDL

Blood haemoglobin level was measured by: (Sahli's Acid haematin method)

\section{Statistical analysis}

The data obtained from various parameters from all groups was analyzed by as per the method suggested by Panse and sukhatme (1967) using Factorial Randomized Block Design (FRBD).

\section{Results and Discussion}

All the results obtained were presented in tabulated format for appropriate analysis. After statistical analysis, data of different groups were presented taking critical difference $(\mathrm{CD})$ at both $5 \%$ and $1 \%$ confidence level. After intra-group and intergroup comparison designations like significant (S), non-significant (NS) and highly significant (HS) were given. 
Superscripts a,b,c... shows significant difference within the column (between different groups on specific day) at $\mathrm{p}=0.05$.
Superscripts $\mathrm{p}, \mathrm{q}, \mathrm{r}$.... shows the significant difference within the row (between different days in a specific group) at $\mathrm{p}=0.05$.

Table.1 Experimental design

\begin{tabular}{|c|l|c|c|}
\hline $\begin{array}{c}\text { Group } \\
\text { N= 12 }\end{array}$ & \multicolumn{1}{|c|}{ Treatment } & \multicolumn{1}{|c|}{ Dose } & Route \\
\hline I & Healthy control & Normal saline as vehicle & - \\
\hline II & Alloxan & $100 \mathrm{mg} / \mathrm{kg}$ body weight & Intraperitonial \\
\hline \multirow{2}{*}{ III } & Alloxan & $100 \mathrm{mg} / \mathrm{kg}$ body weight & Intraperitonial \\
\cline { 2 - 4 } & Metformin & $100 \mathrm{mg} / \mathrm{kg}$ body weight & $\begin{array}{c}\text { Oral in deionised } \\
\text { water }\end{array}$ \\
\hline \multirow{2}{*}{ IV } & Alloxan & $100 \mathrm{mg} / \mathrm{kg}$ body weight & Intraperitonial \\
\cline { 2 - 4 } & $\begin{array}{l}\text { Morinda citrifolia } \\
\text { L. fruit juice }\end{array}$ & $2 \mathrm{ml} / \mathrm{kg}$ body weight & Oral \\
\hline
\end{tabular}

All the animals were maintained for the experimental period of 28 days

Table.2 Blood glucose level (Mean \pm S.E, $\mathrm{mg} / \mathrm{dl}$ ) on different days in experimental rats of different groups

\begin{tabular}{|c|c|c|c|c|c|c|}
\hline \multirow[b]{2}{*}{ Gr. } & \multirow[b]{2}{*}{ Treatment } & \multicolumn{3}{|c|}{ Days } & \multirow[b]{2}{*}{ Stat } & \multirow[b]{2}{*}{ CD } \\
\hline & & $\begin{array}{c}1^{\text {st }} \text { day } \\
(\text { Mean } \pm \text { S.E })\end{array}$ & $\begin{array}{c}14^{\text {th }} \text { day } \\
(\text { Mean } \pm \text { S.E })\end{array}$ & $\begin{array}{c}28^{\text {th }} \text { day } \\
(\text { Mean } \pm \text { S.E })\end{array}$ & & \\
\hline I & Healthy control & $\begin{array}{c}90.43^{\mathrm{dp}} \\
\pm \\
2.84\end{array}$ & $\begin{array}{c}89.29^{\mathrm{dp}} \\
\pm \\
2.73\end{array}$ & $\begin{array}{c}89.18^{\mathrm{dp}} \\
\pm \\
2.03\end{array}$ & NS & \multirow{4}{*}{$\begin{array}{l}\text { At } 5 \% \\
8.65 \\
\\
\text { At } 1 \% \\
11.48\end{array}$} \\
\hline II & $\begin{array}{c}\text { Diabetic } \\
\text { control }\end{array}$ & $\begin{array}{c}422.30^{\mathrm{bpq}} \\
\pm \\
6.77\end{array}$ & $\begin{array}{c}428.79^{\mathrm{ap}} \\
\pm \\
10.05\end{array}$ & $\begin{array}{c}412.66^{\mathrm{aq}} \\
\pm \\
7.25\end{array}$ & $\mathrm{~S}$ & \\
\hline III & $\begin{array}{c}\text { Metformin @ } \\
100 \text { mg/kg BW }\end{array}$ & $\begin{array}{c}443.42^{\text {ap }} \\
\pm \\
9.98 \\
\end{array}$ & $\begin{array}{c}307.90^{\mathrm{bq}} \\
\pm \\
5.79 \\
\end{array}$ & $\begin{array}{c}129.69^{\text {cr }} \\
\pm \\
2.77\end{array}$ & HS & \\
\hline IV & $\begin{array}{c}\text { Morinda } \\
\text { citrifolia } \mathrm{L} . \\
\text { fruit juice @ } \\
2 \mathrm{ml} / \mathrm{kg} \mathrm{BW} \\
\end{array}$ & $\begin{array}{c}396.72^{\mathrm{cp}} \\
\pm \\
7.83\end{array}$ & $\begin{array}{c}287.47^{\mathrm{cq}} \\
\pm \\
8.07\end{array}$ & $\begin{array}{c}155.88^{\mathrm{br}} \\
\pm \\
5.80\end{array}$ & HS & \\
\hline Stat & & HS & HS & HS & & \\
\hline CD & & \multicolumn{3}{|c|}{ At $5 \%-10.0$} & & \\
\hline
\end{tabular}


Table.3 Serum Triglyceride concentration (Mean \pm SE, mg/dl) on different days in experimental rats of different groups

\begin{tabular}{|c|c|c|c|c|c|c|}
\hline \multirow{2}{*}{ Gr. } & \multirow{2}{*}{ Treatment } & \multicolumn{3}{|c|}{ Days } & \multirow{2}{*}{ Stat } & \multirow{2}{*}{ CD } \\
\hline & & $\mathbf{1}^{\text {st }}$ day & $14^{\text {th }}$ day & $28^{\text {th }}$ day & & \\
\hline I & $\begin{array}{l}\text { Healthy } \\
\text { control }\end{array}$ & $\begin{array}{c}93.81^{\mathrm{cp}} \\
\pm \\
3.25\end{array}$ & $\begin{array}{c}93.96^{\mathrm{dp}} \\
\pm \\
2.45\end{array}$ & $\begin{array}{c}90.35^{\mathrm{cp}} \\
\pm \\
3.01\end{array}$ & NS & \multirow{4}{*}{$\begin{array}{c}\text { At } 5 \% \\
6.86 \\
\\
\text { At } 1 \% \\
9.09\end{array}$} \\
\hline II & $\begin{array}{c}\text { Diabetic } \\
\text { control }\end{array}$ & $\begin{array}{c}213.80^{\mathrm{ap}} \\
\pm \\
7.24 \\
\end{array}$ & $\begin{array}{c}217.56^{\mathrm{ap}} \\
\pm \\
5.77 \\
\end{array}$ & $\begin{array}{c}215.27^{\mathrm{ap}} \\
\pm \\
5.95\end{array}$ & NS & \\
\hline III & $\begin{array}{l}\text { Metformin } \\
\text { @ } 100 \\
\text { mg/kg BW }\end{array}$ & $\begin{array}{c}196.15^{\mathrm{bp}} \\
\pm \\
4.60\end{array}$ & $\begin{array}{c}170.94^{\mathrm{bq}} \\
\pm \\
4.37\end{array}$ & $\begin{array}{c}124.91^{\mathrm{br}} \\
\pm \\
2.34\end{array}$ & HS & \\
\hline IV & $\begin{array}{c}\text { Morinda } \\
\text { citrifolia } \mathrm{L} \text {. } \\
\text { fruit juice } \\
\text { @ 2ml/kg } \\
\text { BW } \\
\end{array}$ & $\begin{array}{c}197.03^{\mathrm{bp}} \\
\pm \\
6.74\end{array}$ & $\begin{array}{c}162.38^{\mathrm{cq}} \\
\pm \\
6.02\end{array}$ & $\begin{array}{c}129.66^{\mathrm{br}} \\
\pm \\
5.12\end{array}$ & HS & \\
\hline Stat & & $\mathrm{S}$ & $\mathrm{HS}$ & $\mathrm{HS}$ & & \\
\hline CD & & $\mathrm{At}$ & .92 & 0.50 & & \\
\hline
\end{tabular}

Table.4 Serum total cholesterol concentration (Mean \pm S.E, $\mathrm{mg} / \mathrm{dl}$ ) on different days in experimental rats of different groups

\begin{tabular}{|c|c|c|c|c|c|c|}
\hline \multirow{2}{*}{ Gr. } & \multirow{2}{*}{ Treatment } & \multicolumn{3}{|c|}{ Days } & \multirow{2}{*}{ Stat } & \multirow{2}{*}{ CD } \\
\hline & & $\mathbf{1}^{\text {st }}$ day & $14^{\text {th }}$ day & $28^{\text {th }}$ day & & \\
\hline I & $\begin{array}{l}\text { Healthy } \\
\text { control }\end{array}$ & $\begin{array}{c}85.84^{\mathrm{ap}} \\
\pm \\
3.37\end{array}$ & $\begin{array}{c}85.85^{\mathrm{ap}} \\
\pm \\
3.57\end{array}$ & $\begin{array}{c}85.50^{\mathrm{ap}} \\
\pm \\
3.32\end{array}$ & NS & \multirow{4}{*}{$\begin{array}{l}\text { At } 5 \% \\
3.91 \\
\\
\text { At } 1 \% \\
5.18\end{array}$} \\
\hline II & $\begin{array}{c}\text { Diabetic } \\
\text { control }\end{array}$ & $\begin{array}{c}62.13^{\mathrm{bp}} \\
\pm \\
2.16\end{array}$ & $\begin{array}{c}61.92^{\mathrm{cp}} \\
\pm \\
2.56\end{array}$ & $\begin{array}{c}59.11^{\mathrm{bp}} \\
\pm \\
2.08\end{array}$ & NS & \\
\hline III & $\begin{array}{c}\text { Metformin @ } \\
100 \mathrm{mg} / \mathrm{kg} \\
\mathrm{BW}\end{array}$ & $\begin{array}{c}56.20^{\mathrm{cr}} \\
\pm \\
2.09\end{array}$ & $\begin{array}{c}70.71^{\mathrm{bq}} \\
\pm \\
2.29\end{array}$ & $\begin{array}{c}83.97^{\text {ap }} \\
\pm \\
3.18\end{array}$ & HS & \\
\hline IV & $\begin{array}{c}\text { Morinda } \\
\text { citrifolia L. } \\
\text { fruit juice @ } \\
2 \mathrm{ml} / \mathrm{kg} \mathrm{BW}\end{array}$ & $\begin{array}{c}59.01^{\text {bcr }} \\
\pm \\
3.36\end{array}$ & $\begin{array}{c}74.77^{\mathrm{bq}} \\
\pm \\
3.21\end{array}$ & $\begin{array}{c}87.97^{\text {ap }} \\
\pm \\
3.37\end{array}$ & HS & \\
\hline Stat & & HS & HS & $\mathrm{S}$ & & \\
\hline CD & & \multicolumn{3}{|c|}{ At 5\%-4.52 At 1\%-5.98 } & & \\
\hline
\end{tabular}


Table.5 Serum HDL concentration (Mean \pm S.E, $\mathrm{mg} / \mathrm{dl}$ ) on different days in experimental rats of different groups

\begin{tabular}{|c|c|c|c|c|c|c|}
\hline \multirow{2}{*}{ Gr. } & \multirow{2}{*}{ Treatment } & \multicolumn{3}{|c|}{ Days } & \multirow{2}{*}{ Stat } & \multirow{2}{*}{ CD } \\
\hline & & $1^{\text {st }}$ day & $14^{\text {th }}$ day & $28^{\text {th }}$ day & & \\
\hline I & Healthy control & $\begin{array}{c}29.28^{\mathrm{ap}} \\
\pm \\
1.02\end{array}$ & $\begin{array}{c}29.84^{\mathrm{ap}} \\
\pm \\
0.52\end{array}$ & $\begin{array}{c}29.09^{\mathrm{bp}} \\
\pm \\
0.62\end{array}$ & NS & \multirow{5}{*}{$\begin{array}{l}\text { At } 5 \% \\
2.07 \\
\\
\text { At } 1 \% \\
3.58\end{array}$} \\
\hline II & Diabetic control & $\begin{array}{c}19.81^{\text {bp }} \\
\pm \\
0.84\end{array}$ & $\begin{array}{c}19.84^{\mathrm{cp}} \\
\pm \\
0.49\end{array}$ & $\begin{array}{c}18.97^{\mathrm{cp}} \\
\pm \\
0.95\end{array}$ & NS & \\
\hline III & $\begin{array}{l}\text { Metformin @ } \\
100 \text { mg/kg BW }\end{array}$ & $\begin{array}{c}18.86^{\mathrm{cp}} \\
\pm \\
1.12\end{array}$ & $\begin{array}{c}24.40^{\mathrm{bq}} \\
\pm \\
0.98\end{array}$ & $\begin{array}{c}28.94^{\mathrm{br}} \\
\pm \\
1.13\end{array}$ & HS & \\
\hline IV & $\begin{array}{c}\text { Morinda citrifolia } \\
\text { L. fruit juice @ } \\
2 \mathrm{ml} / \mathrm{kg} \mathrm{BW}\end{array}$ & $\begin{array}{c}22.20^{\mathrm{bp}} \\
\pm \\
3.72\end{array}$ & $\begin{array}{c}30.97^{\mathrm{aq}} \\
\pm \\
3.89\end{array}$ & $\begin{array}{c}41.04^{\mathrm{ar}} \\
\pm \\
4.19\end{array}$ & HS & \\
\hline Stat & & $\mathrm{HS}$ & $\mathrm{HS}$ & $\mathrm{HS}$ & & \\
\hline CD & & \multicolumn{3}{|c|}{ At 5\%-3.13 } & & \\
\hline
\end{tabular}

Table.6 Serum LDL concentration (Mean \pm S.E, mg/dl) on different days in experimental rats of different groups

\begin{tabular}{|c|c|c|c|c|c|c|}
\hline \multirow{2}{*}{ Gr. } & \multirow{2}{*}{ Treatment } & \multicolumn{3}{|c|}{ Days } & \multirow{2}{*}{ Stat } & \multirow{2}{*}{ CD } \\
\hline & & $1^{\text {st }}$ day & $14^{\text {th }}$ day & $28^{\text {th }}$ day & & \\
\hline I & Healthy control & $\begin{array}{c}56.56^{\mathrm{ap}} \\
\pm \\
3.50\end{array}$ & $\begin{array}{c}56.01^{\text {ap }} \\
\pm \\
3.45\end{array}$ & $\begin{array}{c}56.41^{\text {ap }} \\
\pm \\
3.74\end{array}$ & NS & \multirow{5}{*}{$\begin{array}{l}\text { At } 5 \% \\
3.25 \\
\\
\text { At } 1 \% \\
4.30\end{array}$} \\
\hline II & Diabetic control & $\begin{array}{c}42.31^{\mathrm{bp}} \\
\pm \\
2.19\end{array}$ & $\begin{array}{c}42.08^{\mathrm{cp}} \\
\pm \\
2.48\end{array}$ & $\begin{array}{c}40.13^{\mathrm{cp}} \\
\pm \\
2.18\end{array}$ & NS & \\
\hline III & $\begin{array}{l}\text { Metformin @ } \\
100 \text { mg/kg BW }\end{array}$ & $\begin{array}{c}37.33^{\mathrm{cr}} \\
\pm \\
2.13\end{array}$ & $\begin{array}{c}46.30^{\mathrm{bq}} \\
\pm \\
3.00\end{array}$ & $\begin{array}{c}55.03^{\text {ap }} \\
\pm \\
3.50\end{array}$ & HS & \\
\hline IV & $\begin{array}{c}\text { Morinda } \\
\text { citrifolia } \mathbf{L} . \\
\text { fruit juice } @ \\
2 \mathrm{ml} / \mathrm{kg} \mathrm{BW}\end{array}$ & $\begin{array}{c}36.81^{\mathrm{cq}} \\
\pm \\
2.03\end{array}$ & $\begin{array}{c}43.81^{\mathrm{bp}} \\
\pm \\
2.02\end{array}$ & $\begin{array}{c}46.94^{\text {bp }} \\
\pm \\
1.72\end{array}$ & S & \\
\hline Stat & & $\mathrm{HS}$ & $\mathrm{HS}$ & $\mathrm{HS}$ & & \\
\hline CD & & \multicolumn{3}{|c|}{ At $5 \%-3.74$} & & \\
\hline
\end{tabular}


Table.7 Hb values (Mean \pm S.E, g/dl) on different days in experimental rats of different groups

\begin{tabular}{|c|c|c|c|c|c|c|}
\hline \multirow{2}{*}{ Gr. } & \multirow{2}{*}{ Treatment } & \multicolumn{3}{|c|}{ Days } & \multirow{2}{*}{ Stat } & \multirow{2}{*}{ CD } \\
\hline & & $1^{\text {st }}$ day & $14^{\text {th }}$ day & $28^{\text {th }}$ day & & \\
\hline I & Healthy control & $\begin{array}{c}14.31^{\mathrm{ab}} \\
\pm \\
0.51\end{array}$ & $\begin{array}{c}14.00^{\mathrm{ab}} \\
\pm \\
0.40\end{array}$ & $\begin{array}{c}13.66^{\mathrm{b}} \\
\pm \\
0.44\end{array}$ & NS & \multirow{4}{*}{$\begin{array}{l}\text { At } 5 \% \\
0.75 \\
\\
\text { At } 1 \% \\
0.99\end{array}$} \\
\hline II & Diabetic control & $\begin{array}{c}14.48^{\mathrm{ab}} \\
\pm \\
0.69\end{array}$ & $\begin{array}{c}14.40^{\mathrm{ab}} \\
\pm \\
0.69\end{array}$ & $\begin{array}{c}14.53^{\mathrm{a}} \\
\pm \\
0.66\end{array}$ & NS & \\
\hline III & $\begin{array}{l}\text { Metformin @ } \\
100 \text { mg/kg BW }\end{array}$ & $\begin{array}{c}15.00^{\mathrm{a}} \\
\pm \\
0.56\end{array}$ & $\begin{array}{l}14.77^{\mathrm{a}} \\
\pm \\
0.58\end{array}$ & $\begin{array}{c}14.68^{\mathrm{a}} \\
\pm \\
0.58\end{array}$ & NS & \\
\hline IV & $\begin{array}{c}\text { Morinda citrifolia } \\
\text { L. fruit juice @ } \\
2 \mathrm{ml} / \mathrm{kg} \mathrm{BW}\end{array}$ & $\begin{array}{c}13.96^{\mathrm{b}} \\
\pm \\
0.32\end{array}$ & $\begin{array}{c}13.89^{\mathrm{b}} \\
\pm \\
0.33\end{array}$ & $\begin{array}{c}13.71^{\mathrm{b}} \\
\pm \\
0.33\end{array}$ & NS & \\
\hline Stat & & $\mathrm{S}$ & $S$ & $\mathrm{~S}$ & & \\
\hline CD & & \multicolumn{3}{|c|}{ At 5\%-0.87 At 1\%-1.15 } & & \\
\hline
\end{tabular}

After induction of diabetes, serum glucose (Table 2) and triglyceride (Table 3) concentration increases significantly above normal physiological limits. The observations obtained may be due to abnormal carbohydrate and lipid metabolism due to diabetes. However after 28 days of treatment protocol statistically positive results were obtained in all the treatment groups. Our findings were in agreement with the results obtained by Nayak et al., (2011) and Lee et al., (2012).

After induction of diabetes total cholesterol (Table 4), serum HDL (Table 5) and serum LDL (Table 6) concentration decreases significantly below normal physiological limits. Many scientific workers have reported an increase in serum total cholesterol and serum LDL level due to diabetes in humans. However the observations we obtained in wistar rats due to diabetes may be due species variation and in agreement with the results obtained by Ebara et al., (1994) and Pinheiro et al., (2011).

There was no significant change noticed with respect to haemoglobin (Table 7) in all the treated groups. After treatment protocol in group-III, IV gradual improvement in health condition was noted.

In conclusion single intra-peritoneal administration of alloxan monohydrate after overnight fasting can successfully induce diabetes in wistar rats. Due to induction of diabetes, serum glucose and triglyceride level increase and total cholesterol, serum HDL, serum LDL level decreases. There was no significant change noted in respected to haemoglobin level in any of the treated groups. The group which was treated by Morinda citrifolia juice gave better result. But the best result was obtained by the treatment standard anti-diabetic drug i.e. metformin.

\section{References}

Cukierman, T., H.C. Gerstein, Williamson, J.D. 2005. Cognitive decline and dementia in diabetes - systematic overview of prospective observational studies. Diabetologia, 48: 2460-2469.

Ebara, T., T. Hirano, J.C.L. Mamo, R. Sakamaki, S. Furukawa, S. Nagano and Takahashi, T. 1994. Hiperlipidemia in streptozotocin 
diabetic hamster as a model for human insulin deficient diabetes: Comparison to streptozotocin diabetic rats' metabolism. 43: 299-305.

Gardner, D.G. and Shoback, D. 2011. Greenspan's basic and clinical endocrinology, 9th edition, McGraw Hill Education, Newyork, Chaptgr-17, ISBN O-07-162243_8.

Harada, S., W. Fujita-Hamabe, K. Kamiya, S. Tatake and Tokuyama, S. 2010. Involvement of glycemic control in the inhibiting effect of Morinda citrifolia on cerebral ischemia-induced neuronal damage. Yakugaku Zasshi: J. Pharma. Soc. Japan, 130(5): 707-712.

Hirazumi, A., and Furusawa, E. 1999. An immunomodulator Polysaccharide Rich Substance from the Fruit Juice of Morinda Citrifolia (Noni) with Antitumour Activity, Phytother. Res., 13: 380-387.

Kitabchi, A.E., G.E. Umpierrez, J.M. Miles, Fisher, J.N. 2009. Hyperglycemic crises in adult patients with diabetes, Diabetes care, 32: $1335-43$.

Lee, S.Y., S.L. Park, J.T. Hwang, S.H. Yi, Y.D. Nam and Lim, S.L. 2012. Antidiaberic Effect of Morinda citrifolia (Noni) Fermented by Cheonggukjang in KKA(y) Diabetic Mice. Complementary and Alternative Medicine. eCAM, pg 163280.

Nayak, B.S., J.R. Marshall, G. Isitor and Adogwa, A. 2011. Hypoglycemic and Hepatoprotective Activity of Fermented Fruit Juice of Morinda citrifulia (Noni) in Diabetic Rats Complementary and Alternative Medicine, 875293, doi : I 0. I I 551201 I tB7 5293.

O'Brien, R.M. and Granner, D.K. 1996. Regulation of gene expression by insulin. Physiol. Rev., 762: 1109-1161.

O'Gara, P.T., F.G. Kushner, D.D. Ascheim, D.E. Casey, M.K. Chung, J.A. de Lemos, S.M. Ettinger, J.C. Fang, F.M. Fesmire, B.A.
Franklin, C.B. Granger, H.M. Krumholz, J.A. Linderbaum, D.A. Morrow, L.K. Newby, J.P. Ornato, N. Ou, M.J. Radford, J.E. Tamis-Holland, C.L. Tommaso, C.M. Tracy, Y.J. Woo, D.X. Zhao, J.L. Anderson, A.K. Jacobs, J.L. Halperin, N.M. Albert, R.G. Brindis, M.A. Creager, D. DeMets, R.A. Guyton, J.S. Hochman, R.J. Kovacs, F.G Kushner, E.M. Ohman, W.G. Stevenson and Yancy, C.W. 2013. 2013 ACCF/AHA guideline for the management of ST-elevation myocardial infarction: a report of the American College of Cardiology Foundation/American Heart Association Task Force on Practice Guidelines. Circulation, 127: e362-425.

Panse, U.G. and Sukhatme, P.V. 1967. Statistical Methods for Agril. V/orke rs, ICAR Publications, New Delhi.

Pinheiro, L.S., A.D. Melo, A.E. Andreazzi, L.C. Caires, M.B. Costa and Garcia, R.M.G. 2011. Protocol of Insulin Therapy For Streptozotocin-Diabetic Rats Based on a Study of Food Ingestion and Glycemic Variation Scand. J. Lab. Anim, Sci., 3g: 2.

Rivera, A., S. Giono, M. Conzalez, N. Rodriguez and Cedillo, L. 2011. Antibacterial effect of Morinda citrifolia fruit juice against mycoplasmas. Ann. Bio. Res., 491-497.

Sarwar, N., P. Gao, S.R. Seshasai, R. Gobin, S. Kaptoge, E. Di Angelantonio, E. Ingelsson, D.A. Lawlor, E. Selvin, M. Stampfer, C.D. Stehouwer, S. Lewington, L. pennells, A: Thompson, N. Sattar, I.R. White, K.K. Ray and Danesh, J. 2010. Diabetes mellitus, fasting blood glucose concentration, and risk of vascular disease: A collaborative meta-analysis of 102 prospective studies, The Lancet, 375: 2215-22.

Yuankai, S., and Hu, F.B. 2014. The global implications of diabetes and cancer. The Lancet, 383: 1 947-1949.

\section{How to cite this article:}

Nitin D. Jadhav, Debi P. Mishra, Abhinna K. Behera, Sudhir R. Rajurkar and Bhagirath V. Ballurkar. 2017. Studies of Anti-Diabetic Effect of Morinda citrifolia Fruit Juice on Alloxan Induced Diabetic Rat. Int.J.Curr.Microbiol.App.Sci. 6(4): 2021-2028. doi: https://doi.org/10.20546/ijcmas.2017.604.239 